\title{
Aberrant alternative splicing pattern of ADAR2 downregulates adenosine-to-inosine editing in glioma
}

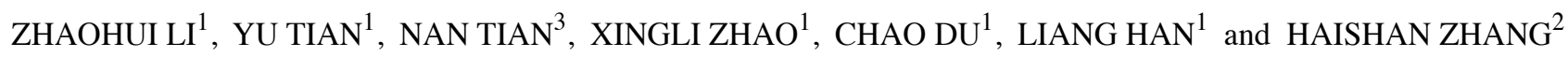 \\ ${ }^{1}$ Department of Neurosurgery and ${ }^{2}$ Surgery Institute, China-Japan Union Hospital of Jilin University, \\ Changchun, Jilin 130033; ${ }^{3}$ Department of Cell Biology, College of Life Science, \\ Zhejiang Chinese Medical University, Hangzhou, Zhejiang 310053, P.R. China
}

Received January 30, 2015; Accepted March 20, 2015

DOI: $10.3892 /$ or.2015.3907

\begin{abstract}
Adenosine-to-inosine (A-to-I) RNA editing is the most common type of RNA editing in mammals, and is catalyzed by adenosine deaminases acting on RNA (ADARs). ADAR2 is the main enzyme responsible for A-to-I editing in humans, and A-to-I underediting at the glutamine (Q)/arginine $(R)$ site of the glutamate receptor subunit B (GluR-B) is associated with the pathogenesis and invasiveness of glioma. The level of ADAR2 mRNA expression and the alternative splicing of the ADAR2 pre-mRNA both affect the catalytic activity of ADAR2. However, reports of ADAR2 mRNA expression in glioma are inconsistent. The mechanism regulating ADAR2 pre-mRNA splicing is also unknown. In this study, we explored the deregulation of A-to-I RNA editing in glioma. We confirmed the underediting at the $\mathrm{Q} / \mathrm{R}$ site of GluR-B mRNA in the glioma cell lines U87, U251 and A172 compared with that in normal human astrocytes (NHAs) HA1800. However, we demonstrated with reverse transcription (RT-PCR) and quantitative PCR (qPCR) that the expression of ADAR2 mRNA was not significantly altered in the glioma cell lines. Three alternative splicing sites are utilized in the glioma cell lines and NHAs: the first, located between exons -1 and 1 , causes the inclusion of exon 1a; the second causes the removal of exon 2, which encodes two double-stranded RNA-binding domains; and the third, located between exons 4 and 6 , causes the inclusion of alternative exon 5a, introducing a 120-nucleotide coding Alu-repeat sequence in frame. However, the expression ratio of two types of transcripts (with and without exon 5a) was altered in the glioma cells. Transcripts with exon 5a, which generate an
\end{abstract}

Correspondence to: Professor Haishan Zhang, Surgery Institute, China-Japan Union Hospital of Jilin University, Changchun, Jilin 130033, P.R. China

E-mail: hszhang@jlu.edu.cn

Abbreviations: A-to-I, adenosine-to-inosine; ADAR, adenosine deaminase acting on RNA; NHAs, normal human astrocytes; GluR-B, glutamate receptor subunit B; PCR, polymerase chain reaction; RT-PCR, reverse transcription-PCR

Key words: glioma, ADAR2, RNA editing, alternative splicing
ADAR2 isoform with $\sim 50 \%$ reduced activity, were predominantly expressed in the glioma cell lines, whereas transcripts without exon 5 a were predominantly expressed in the NHAs. From these results, we conclude that this aberrant alternative splicing pattern of ADAR2 downregulates A-to-I editing in glioma.

\section{Introduction}

RNA editing is a post-transcriptional process that generates many different mRNAs from the same gene. Adenosine-toinosine (A-to-I) RNA editing is the most common type of RNA editing in mammals, and is catalyzed by adenosine deaminases acting on RNA (ADARs). ADAR2 is the main enzyme responsible for A-to-I RNA editing. It has a catalytic domain and three double-stranded RNA binding domains (1-4). The potential impact of RNA editing on the etiology or progression of human diseases is now recognized. Deficient or hyperactive A-to-I RNA editing is associated with several types of human diseases, including epilepsy, amyotrophic lateral sclerosis, immunological disorders and cancers. Analysis of RNA editing in cancers is expected to provide new diagnostic and prognostic markers (5-9).

Gliomas are the most common malignant tumors of the central nervous system, and are almost always fatal. The molecular basis for the malignant progression of glioma involves several collaborative processes. Several studies have shown that a general state of underediting exists in gliomas at several RNA editing sites, including the glutamine (Q)/ arginine (R) site of glutamate receptor subunit B (GluR-B), the $\mathrm{R} / \mathrm{G}$ site of GluR-B, and the $5-\mathrm{HT}_{2 \mathrm{C}}$ serotonin receptor site. Editing the $\mathrm{Q} / \mathrm{R}$ site of GluR-B is essential for the normal function of the neurotransmitter receptor, and underediting at this site is associated with the pathogenesis and invasiveness of glioma $(5,10)$. Significantly reduced editing at the $Q / R$ site has been detected in gliomas and correlates with the grade of the malignancy. A-to-I RNA editing is catalyzed by ADAR2. The level of ADAR2 mRNA expression is thought to play an important role in regulating the editing activity of the enzyme. However, the levels of ADAR2 mRNA expression in gliomas have been inconsistent in previous studies. Furthermore, there is no consistent correlation between the editing efficiency and ADAR2 mRNA expression in those studies. Therefore, 
the regulation of the RNA editing activity of ADAR2 in vivo remains largely unclear (10-13).

Human ADAR2 gene maps to chromosome 21 band q22.3 and spans $\sim 150 \mathrm{~kb}$, and is now known to have 15 exons, as summarized in Fig. 1. Two exons, denoted exons -2 and -1 , specify the 5'-untranslated region (5'-UTR). Exons 0-9 represent the open reading frame that encodes ADAR2. Various ADAR2 transcript isoforms result from multiple alternative splicing events $(3,4)$.

Nine splice sites have so far been detected in human ADAR2 pre-mRNA, as summarized in Table I. Among the possible alternative splicing events, five have been demonstrated to affect the catalytic function of ADAR2 (14-20). In human ADAR2, the inclusion of exon 1a between exon -1 and 1 results in an $\mathrm{N}$-terminal extension of 28 amino acids, which includes seven arginine $(\mathrm{R}) /$ lysine $(\mathrm{K})$ residues. An additional exon, designated exon 0 , positioned between exon -1 and exon 1, has been characterized in human ADAR2. The inclusion of exon 0, initiated at AUG49, results in the addition of 49 amino acids at the $\mathrm{N}$-terminus. Neither the relative protein abundance nor the functional properties of the ADAR2 isoforms containing the altered $\mathrm{N}$-terminal residues have been determined.

Another alternative splicing event in ADAR2 is mediated by A-to-I auto-editing, which creates a new 3' splice acceptor site (changing the adenosine-adenosine to adenosine-inosine) in the intron 1 sequence of the ADAR2 mRNA. This editing-dependent splicing at this site results in the addition of $47 \mathrm{nt}$ to the 5 ' end of exon 2 in the ADAR2 mRNA, causing a frameshift in the coding region. This is predicted to generate a 9-kDa ADAR2 protein because a premature translational termination codon is introduced. Another ADAR2 isoform is produced by alternative splicing in exon 5 , which causes the inclusion of exon 5a. The alternatively spliced exon 5a encodes an in-frame AluJ cassette in the catalytic domain. The inclusion of exon $5 \mathrm{a}$ results in a protein with $\sim 50 \%$ reduced activity. Another alternative splicing event in the human ADAR2 mRNA causes the skipping of exon 2. The lack of exon 2 is predicted to result in a frameshift that would introduce a stop codon in exon 3 , producing a truncated protein of only 12 amino acids if translated.

A newly discovered alternatively spliced isoform of the human ADAR2 gene was reported by Agrant et al (20), in which a 93-nt sequence located in intron 7 is included as exon 7a. The splicing event at this site introduces a premature termination codon and may direct the alternatively spliced transcripts to the nonsense-mediated mRNA decay RNA surveillance pathway. Alternative splicing events between exons 9 and 10 of the human ADAR2 mRNA produce multiple isoforms with different C-terminal sequences or 3'-UTR regions. The use of an alternative splice site within exon 9 of human ADAR2 leads to the replacement of the $29 \mathrm{C}$-terminal residues with two amino acids encoded by exon 10, which generates an inactive protein that cannot edit the GluR-B premRNA. Recent characterization of the human brain ADAR2 transcripts in different developmental stages (fetal, neonatal and adult) revealed four additional C-terminal variants resulting from alternative splicing, which lead to the inclusion of intron 9 and an alternative splicing site in exon 9 at a position $83 \mathrm{nt}$ downstream from the stop codon. Whether

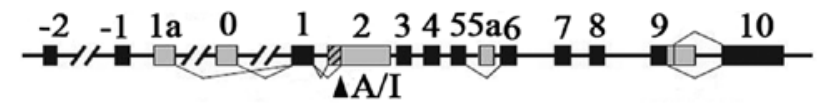

Figure 1. Exon-intron organization of the human ADAR2 gene. Organization of the introns and exons in the ADAR2 gene, which spans $\sim 150 \mathrm{~kb}$ of human chromosome 21q22. Exons are numbered from -2 to 10 and shown as filled boxes; introns and the $5^{\prime}$ and $3^{\prime}$ flanking regions are shown as solid lines. The shaded boxes indicate alternatively utilized exons containing alternative splice sites. Autoediting by ADAR2 within intron 1 creates a 3 ' splice site (from AA to AI), which leads to the insertion of 47 nucleotides.

variations within the 3'-UTR affect the stability, transport, or translational efficiency of ADAR2 requires experimental evidence. The exceptional diversity of the ADAR2 transcripts and consequent ADAR2 protein isoforms implies a physiological mechanism that regulates the functional activities of the ADAR2 enzyme. Alternative splicing is regulated according to the cell type, and the developmental and disease state. The molecular details of how the ADAR2 transcription is regulated in specific tissues or at different developmental stages are poorly understood.

Based on the results of previous studies, we speculated that the level of total ADAR2 mRNA does not necessarily reflect its editing activity in vivo, and that alternative splicing events may regulate the editing activity of human ADAR2 in glioma.

In the present study, we explored the mechanism regulating A-to-I RNA editing in glioma. First, we determined the A-to-I RNA editing level at the Q/R site in the GluR-B mRNA in the human glioma cell lines U87, U251 and A172, and the NHAs HA1800. We then analyzed the expression of ADAR2 mRNA in the glioma cell lines and NHAs, and compared the alternative splicing patterns in these cell lines. We attempt to explain the underediting at the GluR-B Q/R site from the perspectives of the total ADAR2 mRNA expression and the alternative splicing of the ADAR2 pre-mRNA.

\section{Materials and methods}

Cell culture. The human glioma cell lines U87, U251 and A172, and NHAs HA1800 were purchased from Boster Biological Technology, Ltd. (Wuhan, China), and were routinely cultured in Dulbecco's modified Eagle's medium (DMEM) supplemented with $10 \%$ fetal bovine serum (FBS), penicillin $(100 \mathrm{U} / \mathrm{ml})$ and streptomycin $(100 \mu \mathrm{g} / \mathrm{ml})$ in a $5 \% \mathrm{CO}_{2}$ atmosphere at $37^{\circ} \mathrm{C}$. DMEM, FBS, and other tissue culture reagents were purchased from Beijing DingGuo ChangSheng Biotechnology Co., Ltd. (Beijing, China).

The study was approved by the Ethics Committee of the China-Japan Union Hospital of Jilin University, Changchun, Jilin, China.

$R T-P C R$, cloning and sequencing. Total RNA was isolated from the U87, U251, A172, and HA1800 cells using TRIzol reagent (Invitrogen Life Technologies, Carlsbad, CA, USA) and the RNA concentration was determined according to the manufacturer's instructions. Complementary DNA (cDNA) was synthesized using a HiFi-MMLV cDNA kit (Beijing Kang Century Biotechnology Co., Ltd., Beijing, China). 
Table I. Alternative splicing sites in human $A D A R 2$ reported in previous studies.

\begin{tabular}{|c|c|c|c|c|c|}
\hline Authors (ref.) & Year & $\begin{array}{l}\text { Alternative } \\
\text { splice site }\end{array}$ & $\begin{array}{c}\text { Effect on } \\
\text { ADAR2 transcript }\end{array}$ & $\begin{array}{l}\text { Effect on } \\
\text { ADAR2 protein }\end{array}$ & $\begin{array}{l}\text { Effect on } \\
\text { catalytic activity }\end{array}$ \\
\hline Gerber et al (14) & 1997 & $\begin{array}{l}\text { Between } \\
\text { exons } 5 \text { and } 6\end{array}$ & Inclusion of exon $5 \mathrm{a}$ & $\begin{array}{l}\text { Adds an AluJ cassette in } \\
\text { the catalytic domain }\end{array}$ & Reduced \\
\hline Lai et al (15) & 1997 & Within exon 9 & $\begin{array}{l}\text { Truncates the } 3 \text { ' end } \\
\text { of the coding region }\end{array}$ & $\begin{array}{l}\text { Replaces the } 29 \text { C-terminal } \\
\text { residues with } 2 \text { amino acids }\end{array}$ & $\begin{array}{l}\text { No catalytic } \\
\text { activity }\end{array}$ \\
\hline Rueter et al (16) & 1999 & $\begin{array}{l}\text { Between } \\
\text { exons } 1 \text { and } 2\end{array}$ & $\begin{array}{l}\text { Addition of } 47 \text { nucleotides } \\
\text { to the } 5 \text { ' end of exon } 2\end{array}$ & Generates a 9-kDa protein & Reduced \\
\hline $\begin{array}{l}\text { Slavov and } \\
\text { Gardiner (17) }\end{array}$ & 2002 & $\begin{array}{l}\text { Between } \\
\text { exons }-1 \text { and } 1\end{array}$ & Inclusion of exon $1 \mathrm{a}$ & $\begin{array}{l}\text { N-terminal extension by } \\
28 \text { amino acids }\end{array}$ & Unknown \\
\hline Kawahara et al (18) & 2005 & $\begin{array}{l}\text { Between } \\
\text { exons } 1 \text { and } 3\end{array}$ & Skipping of exon 2 & $\begin{array}{l}\text { Produces a protein of only } \\
12 \text { amino acids }\end{array}$ & $\begin{array}{l}\text { No catalytic } \\
\text { activity }\end{array}$ \\
\hline Kawahara et al (18) & 2005 & $\begin{array}{l}\text { Between } \\
\text { exons } 9 \text { and } 10\end{array}$ & Inclusion of intron 9 & Unknown & Unknown \\
\hline Kawahara et al (18) & 2005 & Within exon 9 & $\begin{array}{l}\text { Splices exon } 9 \text { at a position } \\
83 \text { nucleotides downstream } \\
\text { from the stop codon }\end{array}$ & Unknown & Unknown \\
\hline $\begin{array}{l}\text { Mass and } \\
\text { Gommans (19) }\end{array}$ & 2009 & $\begin{array}{l}\text { Between } \\
\text { exons }-1 \text { and } 1\end{array}$ & Inclusion of exon 0 & $\begin{array}{l}\text { N-terminal extension of } \\
49 \text { amino acids }\end{array}$ & Unknown \\
\hline Agranat et al (20) & 2010 & $\begin{array}{l}\text { Between } \\
\text { exons } 7 \text { and } 8\end{array}$ & Inclusion of exon $7 \mathrm{a}$ & $\begin{array}{l}\text { Leads to nonsense-mediated } \\
\text { mRNA decay }\end{array}$ & $\begin{array}{l}\text { No catalytic } \\
\text { activity }\end{array}$ \\
\hline
\end{tabular}

Table II. Primer pairs for RT-PCR.

\begin{tabular}{lll}
\hline Regions & \multicolumn{1}{c}{ Forward } & Reverse \\
\hline Exon -1-Exon 1 & TTCAGGCTGGCATGGAGAGCTT & TGAGGGTTTCTTGACTGGCGGA \\
Exon 1-Exon 4 & CCGCCAGTCAAGAAACCCTCAA & ATGCAAGGCCACGATCACTCAT \\
Exon 4-Exon 6 & TACATGAGTGATCGTGGCCTTGC & GTCCGTAGCTGTCCTCTTGCTT \\
Exon 6-Exon 8 & CAATGCGAGCATCCAAACGTGG & ATGACCTCAATAGCGGAGTCGC \\
Exon 8-Exon 10 & TCAGAGCTTTGAACAGGATACT & GCTGCATGTAGTGGTTCTCC \\
\hline
\end{tabular}

To analyze the alternative splicing of ADAR2 pre-mRNA, five specific primer pairs were designed to amplify different regions that would collectively contain all the alternative splicing sites. The first region is between exon -1 and exon 1 , in which alternative exon 1a is included. Exon 1a has an alternative potential transcription initiation site. The second region includes exons 1-4, and contains two alternative splice sites: one allows the addition of a 47-nt cassette when self-editing occurs in the upstream intron, the other allows exon 2 to be spliced out. The third region covers exons 4-6, and encodes two alternative splice variants. Exon 5 a encodes an in-frame Alu sequence. The fourth region covers exons 6-8, and the inclusion of exon $7 \mathrm{a}$ in this region can lead to nonsense-mediated mRNA decay. The last region includes exons 8-10 and may contain three alternative splice sites. The longest variant contains intron 9. The second longest variant contains the whole of exon 9. In the third longest variant, the latter half of exon 9 is spliced out at a position $83 \mathrm{nt}$ downstream from the stop codon in the long $\mathrm{C}$-terminus. The region encoding the long C-terminus is spliced out from exon 9 in the short variant.
All the primers were designed using Primer 5 software (Premier Biosoft, Palo Alto, CA, USA) and synthesized by GenScript Co., Ltd. (Nanjing, China). The sequences of the primers for ADAR2 amplification are listed in Table II. Primers for the $\beta$-actin gene were 5'-GGCACCACACCT TCTACAAT-3' (forward) and 5'-GGCACCACACCTTCT ACAAT-3' (reverse). PCR was performed using GoldStar Best DNA polymerase (Beijing Kang Century Biotechnology). Reaction conditions were as follows: $9^{\circ} \mathrm{C}$ for $10 \mathrm{~min}$ followed by 40 cycles of degradation at $94^{\circ} \mathrm{C}$ for $30 \mathrm{sec}$, annealing at $56^{\circ} \mathrm{C}$ for $30 \mathrm{sec}$ and extension at $72^{\circ} \mathrm{C}$ for $60 \mathrm{sec}$, and then $72^{\circ} \mathrm{C}$ for $10 \mathrm{~min}$. PCR products were subjected to electrophoresis on a $1.0 \%$ agarose gel, then scanned and analyzed with a gel imaging system. Different bands were all extracted and purified with the Quick Gel Extraction kit, and cloned the PCR products into the pUC-T vector (both from Beijing Kang Century Biotechnology). Positive clones were sequenced by Shanghai Sangon Biological Engineering Technology \& Services Co., Ltd. (Shanghai, China). Similarities between the nucleic acid sequences were based on a two-sequence BLAST analysis. 
Table III. Primer pairs for RT-qPCR.

\begin{tabular}{lll}
\hline Gene/Exons & \multicolumn{1}{c}{ Forward } & \multicolumn{1}{c}{ Reverse } \\
\hline Total ADAR2 & CAATGCGAGCATCCAAACGTGG & ATGACCTCAATAGCGGAGTCGC \\
1a (+) ADAR2 & GGGCAACTGAAGGAGACACACT & GGTGTGCTCATTGGCCATTTCA \\
1a (-) ADAR2 & CGCGGAGTTCTGCTTACTTCAG & GCGGAGACTGTTTCTGTCTTGA \\
$2(+)$ ADAR2 & GCCTGGTTTGCAGTACACACTC & CCTGGTCAGATGTGAAGTCCGT \\
$2(-)$ ADAR2 & GAAAACATGAGTTTTAGCTGACGC & TAATGCAAGGCCACGATCACTC \\
$5 \mathrm{a}(+)$ ADAR2 & TCCTGGAAGGGTCTCGCTCTTAC & GTCCGTAGCTGTCCTCTTGCTT \\
5a $(-)$ ADAR2 & TCCTGGAAGAACCAGCAGATAG & CGAGAAGTAAATGGGCTCCACG \\
$\beta$-actin & GGCACCACACCTTCTACAAT & GGCACCACACCTTCTACAAT
\end{tabular}

Real-time quantitative PCR ( $q P C R$ ) analysis. Primers were designed to amplify the total ADAR2 mRNA and the specific alternative splicing transcripts of ADAR2. All the primers were designed using Primer 5 software and synthesized by GenScript. The sequences of the primers used for real-time PCR are listed in Table III. Total RNA and cDNA were prepared as previously described. RT-qPCR was performed with UltraSYBR mixture (Beijing Kang Century Biotechnology). Reaction conditions were as follows: $95^{\circ} \mathrm{C}$ for $10 \mathrm{~min}$ followed by 40 cycles of degradation at $95^{\circ} \mathrm{C}$ for $15 \mathrm{sec}$, annealing at $56^{\circ} \mathrm{C}$ for $30 \mathrm{sec}$ and extension at $72^{\circ} \mathrm{C}$ for $30 \mathrm{sec}$. All samples were run in triplicate and changes in the gene expression were calculated using the $\Delta \Delta \mathrm{Ct}$ method.

Quantification of $R N A$ editing at the $Q / R$ site of $G l u R-B$. The editing levels at the $Q / R$ site of the GluR-B mRNA were analyzed by RT-PCR and direct sequencing. Total RNA and cDNA were prepared as previously described. Primers for the GluR-B mRNA amplification were 5'-ACCC TTGTGAGAGAGAAGAGGTGAT-3' (forward) and 5'-TGG AGCCAGAGTCTAATGTTCCAT-3' (reverse). An RT-PCR analysis of the region surrounding the $\mathrm{Q} / \mathrm{R}$ site was performed with GoldStar Best DNA polymerase. Reaction conditions were as follows: $95^{\circ} \mathrm{C}$ for $10 \mathrm{~min}$ followed by 40 cycles of degradation at $94^{\circ} \mathrm{C}$ for $30 \mathrm{sec}$, annealing at $57^{\circ} \mathrm{C}$ for $30 \mathrm{sec}$, and extension at $72^{\circ} \mathrm{C}$ for $60 \mathrm{sec}$ and then $72^{\circ} \mathrm{C}$ for $10 \mathrm{~min}$. The PCR products were extracted with the Quick Gel Extraction kit (Beijing Kang Century Biotechnology), and the extracted DNA was sequenced by Shanghai Sangon Biological Engineering Technology and Services. The editing level was calculated from the ratio between the peak areas of the A and $\mathrm{G}$ nucleotides occurring at identical positions in the DNA sequence chromatogram. The ratio between the $\mathrm{A}$ and $\mathrm{G}$ peak heights in individual chromatograms was calculated with FinchTV software (Geospiza Inc., Seattle, WA, USA). The percentage editing was calculated from the peak heights as: $(G /(A+G)] \times 100$.

Statistical analysis. SPSS 16.0 statistical software (SPSS Inc., Chicago, IL, USA) was used for the statistical analysis. Data are expressed as means \pm standard deviations (SD) of experiments performed in triplicate. Statistical analyses were performed with one-way analysis of variance (ANOVA) for multiple comparisons and the Student's t-test for the comparison of groups. $\mathrm{P}<0.05$ was considered to indicate a statistically significant difference.

\section{Results}

$Q / R$ site of GluR-B mRNA is underedited in the human glioma cell lines. We analyzed the editing status at the $\mathrm{Q} / \mathrm{R}$ site of GluR-B mRNA in the glioma-derived cell lines U87, U251 and A172, and the normal control HA1800 cells. A GluR-B cDNA fragment covering the Q/R site was PCR-amplified from reverse-transcribed total RNA. We directly sequenced the GluR-B PCR amplicons. The genomically encoded glutamine $(\mathrm{Q})$ codon, $\mathrm{CAG}$, was edited to an arginine (R) codon (appearing as CGG in cDNA) in $100 \%$ of the molecules in the normal HA1800 astrocytes. In contrast, all the glioma cell lines showed a reduced level of editing at the $\mathrm{Q} / \mathrm{R}$ site, indicated by mixed $\mathrm{A}$ and $\mathrm{G}$ signals at the $\mathrm{Q} / \mathrm{R}$ site. The A-to-I RNA editing levels in the U87, U251 and A172 cells were 92, 89 and 83\%, respectively (Fig. 2).

Expression of total ADAR2 $\mathrm{mRNA}$ is not significantly altered in the human glioma cell lines. To investigate the mechanism underlying the differences in the editing levels in the glioma cell lines and NHAs, we assessed the expression of ADAR2 mRNA. Using RT-qPCR, we found that the expression of ADAR2 mRNA was not significantly altered in the glioma cell lines U87, U251 and A172 compared with that in the NHAs HA1800 (Fig. 3).

Three alternative splicing sites in ADAR2 pre-mRNA were detected in human glioma cell lines and NHAs. The whole $A D A R 2$ cDNA was divided into five overlapping fragments, collectively containing all the alternative splicing sites. Five specific primer pairs were designed to amplify the different regions. The PCR products were analyzed with agarose gel electrophoresis, and then cloned and sequenced. When the primers amplifying the region covering exon -1 and 1 were used, one band joining exon -1 and 1 , and a longer additional band were amplified in all the cell lines. When the primers amplifying the region covering exon 1 and 4 were used, one band joining exons $1,2,3$, and 4 , and a shorter additional band were amplified. When the primers amplifying the region between exon 4 and 6 were used, one band joining exons 4, 5 and 6, and a longer additional band were amplified. When the primers amplifying the region covering exon 6 and 8 were used, only one band was amplified, and when the primers amplifying the region covering exon 8 and 10 were used, only one band was amplified (Fig. 4A). Sequencing 


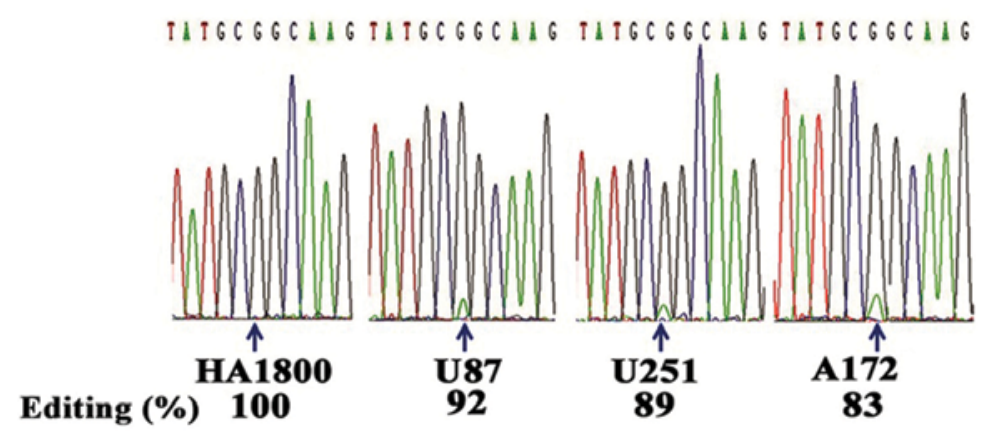

Figure 2. A-to-I editing levels at the Q/R site of GluR-B mRNA in human glioma cell lines and NHAs. The results shown are for the codon encoding the $\mathrm{Q} / \mathrm{R}$ site (marked with arrow) and its neighboring codons. The percentage editing is indicated below. A-to-I, adenosine-to-inosine; NHAs, normal human astrocytes; GluR-B, glutamate receptor subunit B.

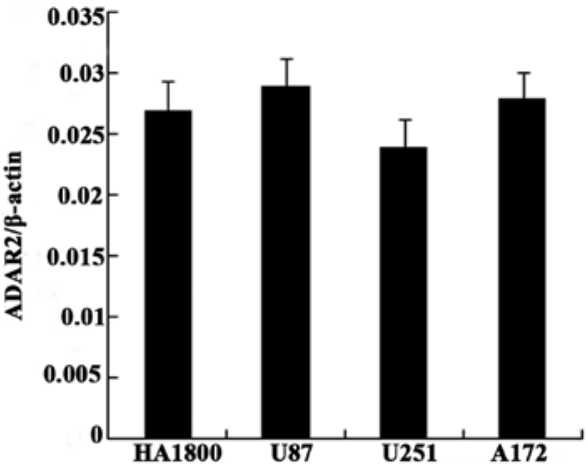

Figure 3. Expression levels of total ADAR2 mRNA in the glioma cell lines and NHAs. The fold expression is shown in arbitrary units, calculated as the relative change in expression normalized to the expression of $\beta$-actin mRNA. Error bars indicate the standard deviations of triplicate experiments. NHAs, normal human astrocytes.

showed that all the bands corresponded to some specific fragment of the ADAR 2 mRNA. Among these bands, the longer product between exon -1 and exon 1 contained an additional 47-nt exon, which was identical to exon 1a (Fig. 4B). The shorter product between exon 1 and 4 lacked a 935-nt exon corresponding to exon 2 (Fig. 4C), and the longer fragment between exon 4 and 6 contained an additional 120-nt exon identical to exon 5a (Fig. 4D).

RT-PCR and sequencing showed that three alternative splice sites in the ADAR2 mRNA were utilized in both the human glioma cell lines and the NHAs. We designated these alternative splice sites ASS1, ASS2 and ASS3. ASS1 is located between exon -1 and 1 . Alternative splicing at this site creates exon 1a, the inclusion of which results in an alternative potential transcription initiation site and an N-terminal extension of 28 amino acids. ASS2 is located between exon 1 and 3. Alternative splicing at ASS2 removes the whole of exon 2, resulting in a frameshift that introduces a downstream stop codon. ASS3 is located between exon 4 and 6 . Alternative splicing at this site involves the inclusion of alternative exon 5a, which introduces an in-frame 120-nt Alu-repeat-encoding sequence. No other previously reported alternative splicing sites in $A D A R 2$ were utilized in the human glioma cell lines or NHAs.

Unbalanced expression among alternatively spliced ADAR2 transcripts in human glioma cell lines. Since three alterna- tive splice sites in the ADAR2 pre-mRNA are spliced in the human glioma cell lines and NHAs, the downregulated catalytic activity of ADAR2 in gliomas cannot be simply attributed to differences in the alternative splice sites utilized. Therefore, we investigated whether the expression ratios of the different ADAR2 transcripts produced by alternative splicing are disturbed in the human glioma cell lines.

Alternative splicing yields two types of transcripts at each of the three alternative splice sites. Alternative splicing at ASS1 produces ADAR2 transcripts with and without exon 1a, designated exon 1a (+) and 1a (-), respectively. Similarly, alternative splicing at ASS2 produces two transcripts designated exon $2(+)$ and $2(-)$, and alternative splicing at ASS3 produces two transcripts, designated exon 5a (+) and 5a (-).

To investigate the expression ratios of the different ADAR2 transcripts produced by specific alternative splicing events, we analyzed the products of each of the three alternative splice sites independently. A quantitative analysis was performed with RT-qPCR. Two primer pairs were designed complementary to each alternative splice site. To amplify the exon 1a (+) transcripts, the sense primer was located in exon -1 and the antisense primer in exon 1a. For the exon 1a (-) transcripts, the sense primer was located in exon -1 , and the antisense primer flanked the exon $-1 /$ exon 1 splice junction. RT-qPCR revealed that the relative expression levels of exon 1a (-)/exon 1a (+) did not differ between the human glioma cell lines and NHAs (Fig. 5A). Similarly, the sense primer for exon $2(+)$ was located in exon 2 and the antisense primer was located in exon 4 . The sense primer for exon 2 (-) flanked the exon 2/exon 3 splice junction and the antisense primer was located in exon 4 . There was no significant difference in the exon $2(-) /$ exon $2(+)$ expression levels in the glioma cell lines and NHAs (Fig. 5B).

At the third alternative splice site, ASS3, the sense primer for exon $5 \mathrm{a}(+)$ was located at the exon 5a/exon 6 splice junction and the antisense primer flanked exon 8 . The sense primer for exon 5a (-) flanked the exon 5/exon 6 splice junction and the antisense primer was located in exon 8 . With RT-qPCR, we demonstrated that the exon 5a (-)/exon 5a (+) ratio in the human glioma cell lines was lower than that in the NHAs. Therefore, in the glioma cell lines, the exon 5a (+) transcripts were predominantly expressed, whereas the exon 5a (-) transcripts were predominantly expressed in the NHAs (Fig. 5C). 

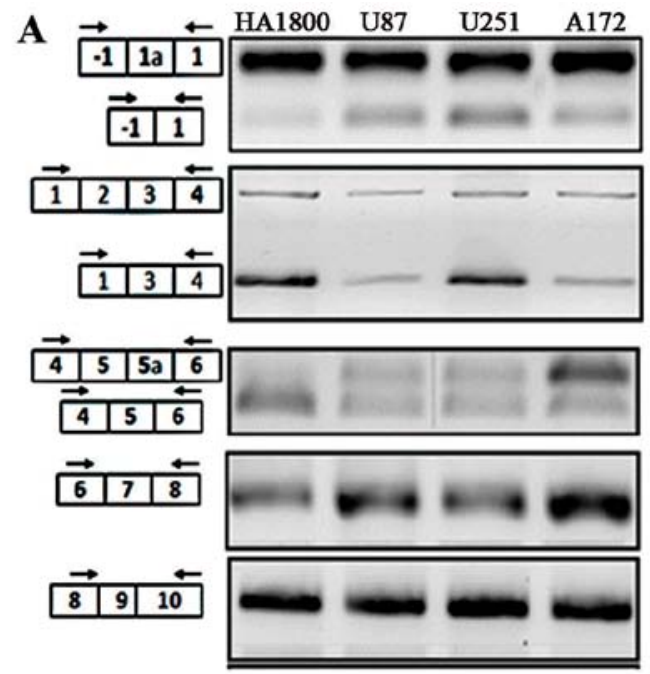

B
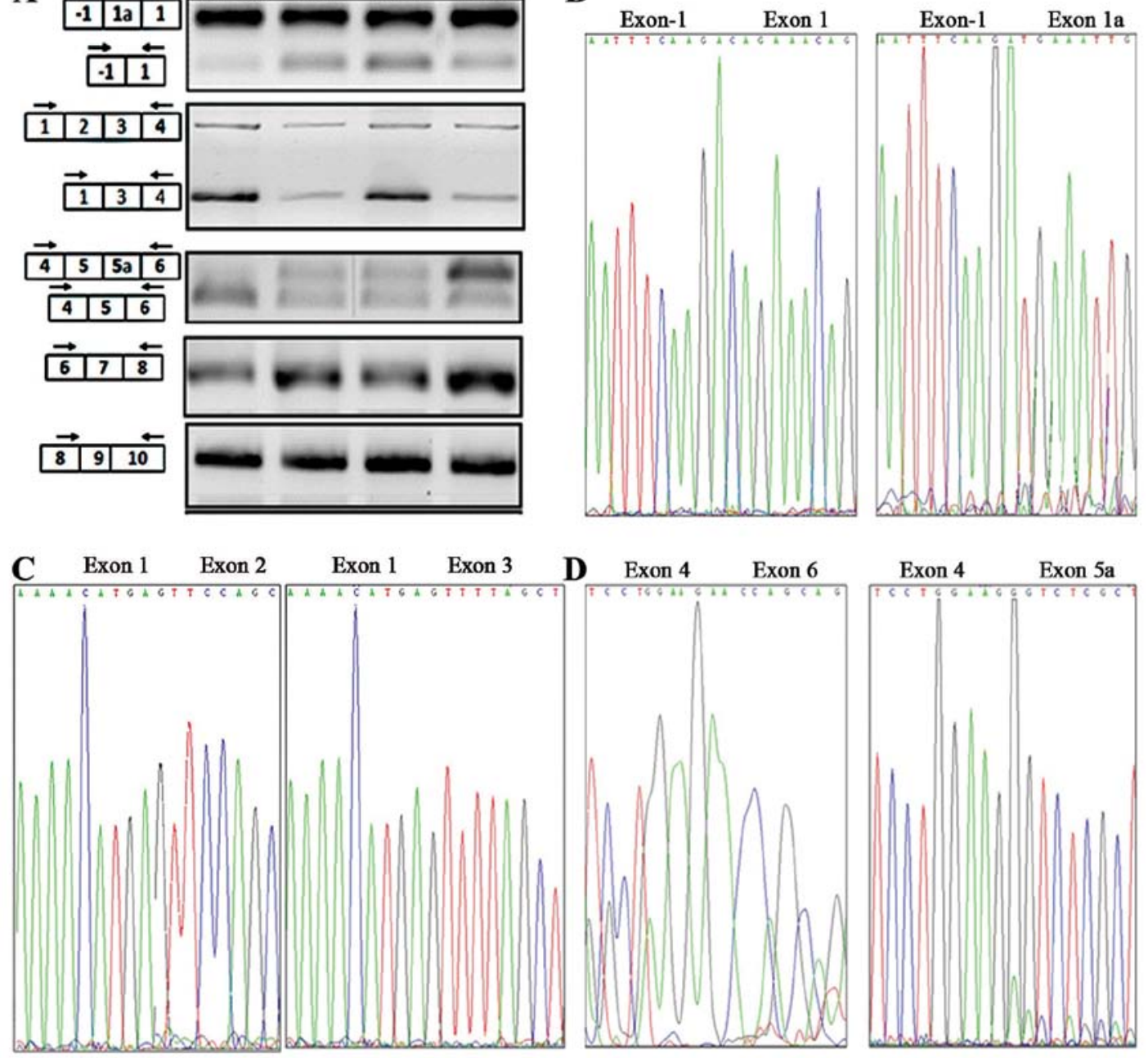

Figure 4. Three alternative splicing sites in ADAR2 pre-mRNA are utilized in both the glioma cell lines and NHASs. (A) Agarose gel electrophoresis result of ADAR2 PCR amplicons, which were amplified using five specific primer pairs collectively containing all the alternative splicing sites. (B) Sequence analysis of the PCR product amplified between exon -1 and exon 1. Shown is the electropherogram of the normal band and the longer band including exon 1a. (C) Sequence analysis of the PCR product amplified between exon 1 and exon 4. Shown is the electropherogram of the normal band and the shorter band lacking exon 2. (D) Sequence analysis of the PCR product amplified between exon 4 and exon 6 . Shown is the electropherogram of the normal band and the longer band containing exon $5 \mathrm{a}$. NHAs, normal human astrocytes.

\section{Discussion}

Gliomas are the most aggressive and lethal tumors of the central nervous system. In contrast to patients with other types of cancer, the outcomes of patients with glioma have only marginally improved in the past decades mainly due to the lack of a comprehensive understanding of the molecular mechanisms underlying glioma. A-to-I RNA editing is an essential post-transcriptional modification in the brain and varies little among healthy individuals. However, deregulated RNA editing has been found in many different human cancers, including glioma. Many studies have shown that the $Q / R$ site of the GluR-B mRNA is underedited in gliomas compared with its editing in normal tissue. The A-to-I editing catalyzed by ADAR 2 at this site is essential for reducing the invasiveness of glioma, and its dysregulation may be crucial in glioma pathogenesis. Galeano et al (21) demonstrated that the editing activity of ADAR2 inhibits glioblastoma growth by modulating the CDC14B/SKP2/p21/p27 axis. In this study, we analyzed the editing status of the GluR-B Q/R site in the glioma cell lines
U87, U251 and A172, and in NHAs. The editing levels at the $\mathrm{Q} / \mathrm{R}$ site were reduced in all three glioma cell lines compared with that in NHAs, confirming that ADAR2-mediated A-to-I editing is significantly reduced in gliomas.

ADAR2 is the enzyme responsible for A-to-I editing at the Q/R site of the GluR-B mRNA. Two factors may account for the reduced A-to-I editing in gliomas. First, the levels of ADAR2 mRNA expressed may be reduced in glioma. However, recent findings regarding ADAR2 mRNA expression in gliomas have been inconsistent. The first study by Maas et al (10) showed that ADAR2 mRNA expression was not significantly altered in one oligodendroglioma and seven glioblastoma multiforme samples, whereas Paz et al (11) observed a pronounced reduction in ADAR2 mRNA in gliomas of different grades in an analysis of 18 brain tumors. Cenci et al (12) analyzed 14 astrocytoma tissue samples from 10 children, and found no significant difference in the ADAR2 mRNA levels in the tumor tissues and normal white matter. The other possible explanation is that the catalytic activity of ADAR2 is abnormal. ADAR2 pre-mRNA undergoes a number of well-known 

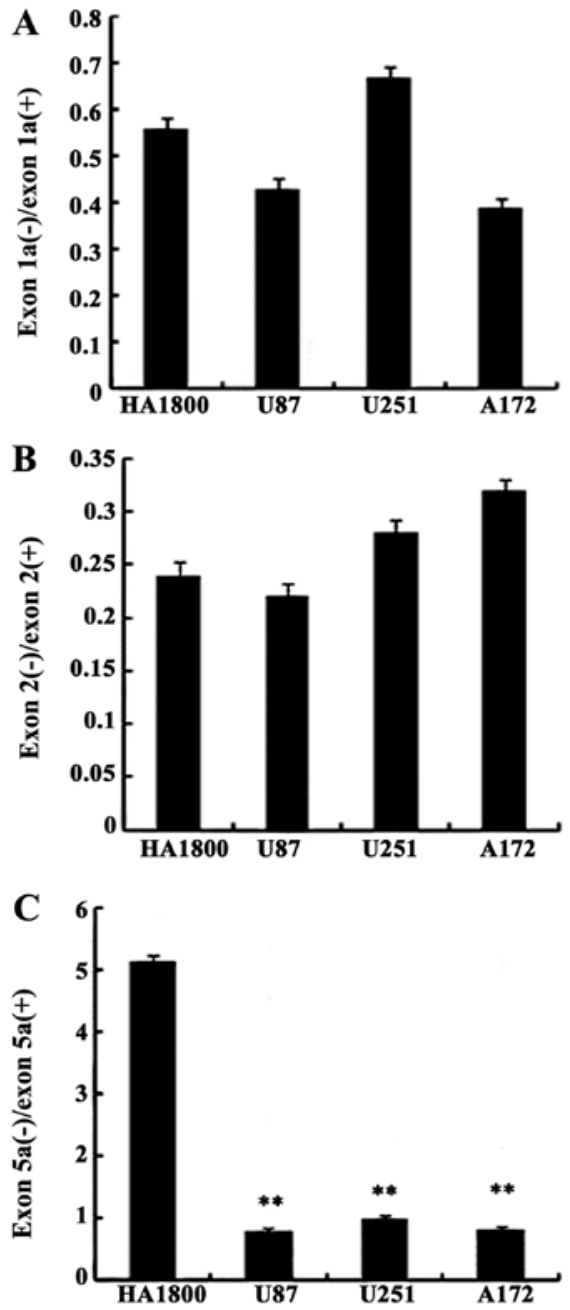

Figure 5. Relative expression of the ADAR2 transcripts produced by alternative splicing at the three alternative splicing sites, analyzed with RT-qPCR. Each of the three alternative splice sites was analyzed independently. (A) The relative expression of exon 1a (-)/exon 1a (+) did not differ between the human glioma cell lines and NHAs. (B) There was no significant difference between the relative expression of exon $2(-) /$ exon $2(+)$ in the glioma cell lines and NHAs. (C) The exon 5a (-)/ exon 5a (+) ratio was lower in the human glioma cell lines than that in the NHAs. ${ }^{* *} \mathrm{P}<0.01$ vs. HA1800. Error bars indicate the standard deviation of triplicate experiments. NHAs, normal human astrocytes.

alternative splicing events, some affecting its function. A combination of genetic and epigenetic factors influences the precise cell-type- and developmental-stage-specific selection of alternative splice sites. The mechanisms underlying the regulation of both the expression and the enzymatic activity of human ADAR2 remain unknown. There is little knowledge of the mechanisms that regulate RNA editing in vivo. Therefore, the characterization of ADAR expression and the identification of alternative ADAR2 variants is an important prerequisite for understanding the mechanisms that regulate RNA editing and deregulate it in diseases.

To identify the factors that are responsible for the reduced RNA-editing activity of ADAR2, we first examined the expression of ADAR2 mRNA in the human glioma cell lines and NHAs using RT-qPCR. We found no differences in the ADAR2 mRNA expression in these cell types. Our results are consistent with most studies that have analyzed ADAR 2 mRNA expression. Based on the results of our study and previous reviews, we consider that the expression of ADAR2 mRNA is not significantly altered in glioma and that the underediting at the $\mathrm{Q} / \mathrm{R}$ site of GluR-B in glioma is not attributable to altered ADAR2 expression. Therefore, we speculated that the underediting at the $Q / R$ site of GluR-B mRNA is attributable to the abnormal catalytic activity of ADAR 2 caused by alternative splicing.

Alternative splicing is a key molecular event that increases protein diversity. Through this process, the coding capacity of a single gene is increased and several related proteins with diverse and even antagonistic functions are expressed. Aberrant splicing is associated with various diseases, including cancer. Increasing evidence indicates that cancer-associated splicing variants play an important role in tumor initiation and progression. Changes in the normal process of alternative splicing in cancer cells cause the production of novel mRNAs or the modification of the tissue-specific ratios between normal mRNA isoforms. Although most aberrant mRNAs are degraded by the nonsense-mediated mRNA decay pathway, some new mRNA species can reduce the levels of normal protein or give rise to different protein isoforms with potentially tumorigenic properties. Several reviews have provided lists of many genes that display cancer-related splicing changes. The alternative splicing of cancer-related genes could affect cell-cycle control, signal transduction pathways, apoptosis, angiogenesis, invasion and metastasis (22-27).

To investigate whether alternative splicing plays a role in regulating the catalytic activity of ADAR2 and affects A-to-I editing at the $\mathrm{Q} / \mathrm{R}$ site of GluR-B mRNA in gliomas, we analyzed the alternative splicing patterns of ADAR 2 in the glioma cell lines and NHAs.

As the first step, we analyzed the alternative splicing sites in the ADAR2 pre-mRNA in human glioma cells and NHAs. Five specific primer pairs were designed to amplify different regions of the $A D A R 2$ cDNA, which collectively contained all the alternative splicing sites. The PCR products were analyzed with agarose gel electrophoresis and then cloned and sequenced. The RT-PCR and sequencing results showed that three alternative splicing sites were utilized in both the human glioma cell lines and NHAs. ASS1 is located between exon -1 and exon 1. Alternative splicing at this site creates exon 1a, the inclusion of which results in an alternative potential transcription initiation site and an N-terminal extension of 28 amino acids. ASS2 is located between exon 1 and 3. Alternative splicing at ASS2 removes the whole exon 2 and produces a frameshift that introduces a downstream stop codon. ASS3 is located between exon 4 and 6 . Alternative splicing at this site involves the inclusion of alternative exon 5a, which introduces an in-frame 120-nt Alu-repeat encoding sequence. No utilization of the other previously reported alternative splice sites in $A D A R 2$ was detected in the human glioma cell lines or NHAs. The alternative splice sites in ADAR2 utilized in the glioma cell lines were identical to those utilized in NHAs.

We also investigated whether the expression ratios of different ADAR2 transcripts differed between the glioma cell lines and NHAs. The expression ratios of the different transcripts of ADAR2 generated by alternative splicing at each of the three splicing sites were measured with RT-qPCR. The relative expression levels of exon 1a (-)/exon 1a (+) did 
not differ between the human glioma cell lines and NHAs. Similarly, the relative expression of exon $2(-) /$ exon $2(+)$ did not differ significantly between the glioma cell lines and NHAs. However, the exon 5a (-)/exon 5a (+) ratio was lower in the human glioma cell lines than that in the NHAs. Thus, in the glioma cell lines, the exon $5 \mathrm{a}(+)$ transcripts predominated, whereas the exon $5 \mathrm{a}(-)$ transcripts predominated in the NHAs.

Changes in the normal alternative splicing processes in cancer cells result in the production of novel mRNAs or in the modification of the tissue-specific ratios between the normal mRNA isoforms. In this study, we found that the alternatively splice sites utilized in the glioma cell lines and NHAs were identical. However, the expression ratios between the normal mRNA isoforms generated by splicing at ASS3 differed in the two types of cells. However, whether the altered forms caused the cancer or are merely innocent bystanders generated by the changes in the mRNA processing machinery remains unknown.

We investigated, for the first time, the underediting of GluR-B Q/R by ADAR2 in glioma from the perspectives of the total ADAR2 mRNA expression and the alternative splicing of ADAR2 pre-mRNA. We found that the total ADAR2 mRNA expression did not differ between the glioma cell lines and NHAs and that the same three alternative splicing sites were used in both the glioma cell lines and NHAs. However, the relative expression of the different transcripts differed in the glioma and normal cells. In the glioma cell lines, exon $5 \mathrm{a}(+)$ transcripts, which generate an ADAR2 isoform with $\sim 50 \%$ reduced activity, predominated. Thus, we conclude that an aberrant alternative splicing pattern in the ADAR2 pre-mRNA contributes to the downregulation of A-to-I editing in glioma.

\section{Acknowledgements}

The authors thank Professor Chunlei Fan for technical assistance. The present study was supported by grants from the National Science Foundation of China (no. 30672159) and New Century Excellent Talents in Chinese Universities (NCET-06-0306).

\section{References}

1. Tang W, Fei Y and Page M: Biological significance of RNA editing in cells. Mol Biotechnol 52: 91-100, 2012.

2. Jacobs MM, Fogg RL, Emeson RB and Stanwood GD: ADAR1 and ADAR2 expression and editing activity during forebrain development. Dev Neurosci 31: 223-237, 2009.

3. Goodman RA, Macbeth MR and Beal PA: ADAR proteins: structure and catalytic mechanism. Curr Top Microbiol Immunol 353: 1-33, 2012.

4. George CX, Gan Z, Liu Y and Samuel CE: Adenosine deaminases acting on RNA, RNA editing, and interferon action. J Interferon Cytokine Res 31: 99-117, 2011.

5. Kubota-Sakashita $\mathrm{M}$, Iwamoto $\mathrm{K}$, Bundo $\mathrm{M}$ and Kato T: A role of ADAR2 and RNA editing of glutamate receptors in mood disorders and schizophrenia. Mol Brain 7: 5, 2014.
6. Slotkin W and Nishikura K: Adenosine-to-inosine RNA editing and human disease. Genome Med 5: 105-112, 2013.

7. Avesson L and Barry G: The emerging role of RNA and DNA editing in cancer. Biochim Biophys Acta 1845: 308-316, 2014.

8. Galeano F, Tomaselli S, Locatelli F and Gallo A: A-to-I RNA editing: The 'ADAR' side of human cancer. Semin Cell Dev Biol 23: 244-250, 2012.

9. Dominissini D, Moshitch-Moshkovitz S, Amariglio N and Rechavi G: Adenosine-to-inosine RNA editing meets cancer. Carcinogenesis 32: 1569-1577, 2011.

10. Maas S, Patt S, Schrey M and Rich A: Underediting of glutamate receptor GluR-B mRNA in malignant gliomas. Proc Natl Acad Sci USA 98: 14687-14692, 2001.

11. Paz N, Levanon EY, Amariglio N, Heimberger AB, Ram Z, Constantini S, Barbash ZS, Adamsky K, Safran M, Hirschberg A, et al: Altered adenosine-to-inosine RNA editing in human cancer. Genome Res 17: 1586-1595, 2007.

12. Cenci C, Barzotti R, Galeano F, Corbelli S, Rota R, Massimi L, Di Rocco C, O'Connell MA and Gallo A: Down-regulation of RNA editing in pediatric astrocytomas: ADAR2 editing activity inhibits cell migration and proliferation. J Biol Chem 283: 7251-7260, 2008.

13. Tomaselli S, Galeano F, Massimi L, Di Rocco C, Lauriola L, Mastronuzzi A, Locatelli F and Gallo A: ADAR2 editing activity in newly diagnosed versus relapsed pediatric high-grade astrocytomas. BMC Cancer 13: 255-260, 2013.

14. Gerber A, O'Connell MA and Keller W: Two forms of human double-stranded RNA-specific editase 1 (hRED1) generated by the insertion of an Alu cassette. RNA 3: 453-463, 1997.

15. Lai F, Chen CX, Carter KC and Nishikura K: Editing of glutamate receptor B subunit ion channel RNAs by four alternatively spliced DRADA2 double-stranded RNA adenosine deaminases. Mol Cell Biol 17: 2413-2424, 1997.

16. Rueter SM, Dawson TR and Emeson RB: Regulation of alternative splicing by RNA editing. Nature 399: 75-80, 1999.

17. Slavov D and Gardiner K: Phylogenetic comparison of the pre-mRNA adenosine deaminase ADAR2 genes and transcripts: conservation and diversity in editing site sequence and alternative splicing patterns. Gene 299: 83-94, 2002.

18. Kawahara Y, Ito K, Ito M, Tsuji S and Kwak S: Novel splice variants of human ADAR 2 mRNA: skipping of the exon encoding the dsRNA-binding domains, and multiple C-terminal splice sites. Gene 363: 193-201, 2005.

19. Maas S and Gommans WM: Novel exon of mammalian ADAR2 extends open reading frame. PLoS One 4: e 4225, 2009.

20. Agranat L, Sperling J and Sperling R: A novel tissue-specific alternatively spliced form of the A-to-I RNA editing enzyme ADAR2. RNA Biol 7: 253-262, 2010.

21. Galeano F, Rossetti C, Tomaselli S, Cifaldi L, Lezzerini M, Pezzullo M, Boldrini R, Massimi L, Di Rocco CM, Locatelli F, et al: ADAR2-editing activity inhibits glioblastoma growth through the modulation of the CDC14B/Skp2/p21/p27 axis. Oncogene 32: 998-1009, 2013.

22. Kim YJ and Kim HS: Alternative splicing and its impact as a cancer diagnostic marker. Genomics Inform 10: 74-80, 2012.

23. Zhang J and Manley JL: Misregulation of pre-mRNA alternative splicing in cancer. Cancer Discov 3: 1228-1237, 2013.

24. Oltean S and Bates DO: Hallmarks of alternative splicing in cancer. Oncogene 33: 5311-5318, 2014.

25. Sette $C$, Ladomery $M$ and Ghigna $C$ : Alternative splicing: role in cancer development and progression. Int J Cell Biol 2013: 421606, 2013.

26. Bonomi S, Gallo S, Catillo M, Pignataro D, Biamonti G and Ghigna C: Oncogenic alternative splicing switches: role in cancer progression and prospects for therapy. Int J Cell Biol 2013: 962038 , 2013.

27. Liu S and Cheng C: Alternative RNA splicing and cancer. Wiley Interdiscip Rev RNA 4: 547-566, 2013. 